\title{
A New Model For Induction Motor With Induced Saliencies
}

\author{
Edinson Franco IEEE Member, Martha Amaya and José Ramírez, IEEE Member.
}

\begin{abstract}
This paper develops a formal and general model for an induction motor with periodic variations of the rotor slot; this induction motor with induced saliencies is commonly used in sensorless control applications. In order to obtain the model, first the analysis of the stator and rotor inductances modified by induced saliencies is carried out; second, the electromagnetic and electomechanical equations in $\mathrm{ABC}$ frame are developed and third, the model is represented in the natural orthogonal a-b frames. The obtained model is suitable for control design and compatible with the classical ab model for induction motors without saliencies.
\end{abstract}

Index Terms - Induction motor, induced saliencies, sensorless control.

$\begin{array}{ll}\text { Variables } & \text { NOMENCLATURE } \\ i & : \text { electrical current } \\ \Psi, \psi & : \text { magnetic flux } \\ \mathcal{F} & : \text { magnetomotive force } \\ B & : \text { flux densities } \\ \alpha, \theta_{r} & : \text { rotor angle, }\left(\alpha=\nu \theta_{r}\right) \\ T_{l} & : \text { mechanical load torque } \\ T_{e} & : \text { electrical torque } \\ \text { Parameters } & \\ \nu & : \text { number of pole-pairs } \\ R & : \text { electrical resistence } \\ L & : \text { electrical inductance } \\ M & : \text { inertial mass } \\ f & : \text { friction coefficient } \\ \mu_{0} & : \text { the air magnetic permeability } \\ l & : \text { the axial length of the machine } \\ r & : \text { mean radius at the air gap of the machine } \\ \text { Superscripts } & : \text { conventional three-axis systems } \\ \text { ABC } & : \text { orthogonal three-axis systems } \\ \text { abh } & : \text { orthogonal by-axis systems } \\ \text { ab } & : \text { refer to the stator frame } \\ \text { Subscripts } & \text { : the the rotor frame } \\ \mathrm{s} & \text { : }\end{array}$

Amaya M., and Ramirez J. are teachers at the Electrical and Electronic Engineering School of the "Universidad del Valle", Calle 13 No.100-00 Cali, Colombia.

J. Ramírez and E. Franco works with Industrial Control Research Group. jomirameunivalle.edu.co efmeunivalle.edu.co

Martha Amaya work with the Energy Conversion Research Group. martha. amaya@univalle.edu.co

\section{INTRODUCTION}

In the past and present decade, the control for Induction Motors (IM) without mechanical shaft sensors has led to researchers and manufacturers to include sensorless vector drives.

The main problem in the sensorless control of induction motors is around zero speed or zero excitation frequency. In order to solve the problem, several works have been developed for modelling the phenomena in the stator and rotor slots; other ones more complexes, include a detailed tensor model of the IM; however, these approaches are something complex for control.

The design of high performance sensorless drives of induction motors include an observer for the rotor/stator flux and one estimation function for the mechanical speed; some problems have been reported and can be summarized as: loss of observability around zero excitation frequency, incorrect flux/torque estimation induced by errors in the estator and rotor resistances, steady-state inestability at low speed, particularly under regeneration. About solving the loss of observability at zero excitation frequency, one possible alternative solution is the Induction Motor with Induced Saliencies IMIS.

The saliencies of the IM used for sensorless control have been classified as: slot harmonics, designed asymetry (induced saliencies), saturation and dynamic eccentricity. The induced saliencies can be described as changes in the rotor slots geometry.

The sensorless control of the IMIS, for position tracking and speed control including both saliencies and input high frequency signals, has been reported by Holtz [1], [2], Degner[3] and Jansen [4] with promising results; Quan[5] has reported the high performance near to zero speed.

In this paper only changes in the rotor slots width are considered, then for obtain the model, the flux variations induced by the saliencies must be took into account; other important assumptions are: a) the variation of the rotor slots width can be represented for a periodical (with a pole pitch) and sinusoidal permeance; b) the squirrel-cage motor is approximated by an equivalent polyphase wound rotor with the same pole number and equivalents turns $\mathrm{Nr}$ and resistance $\mathrm{Rr}$; c) the stator and rotor windings may be approximated as sinusoidal distributed windings; d) the stator and rotor steel have a high permeability; and e) the air gap is assumed uniform and the Carter factor is modified to approach the saliencies phenomena. 


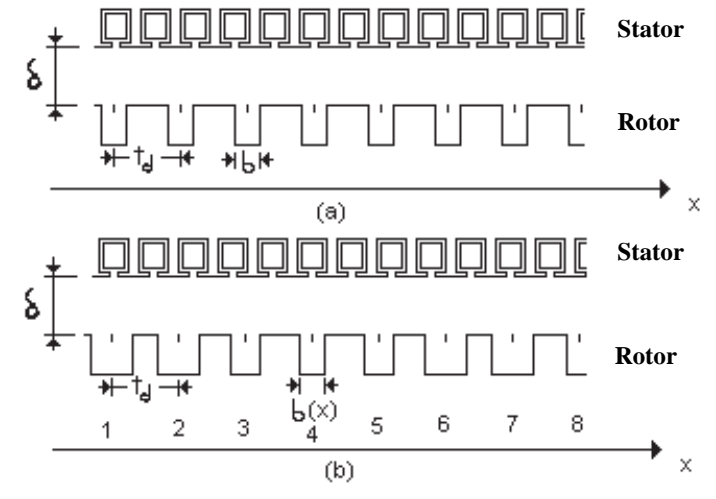

Fig. 1. IM stator and rotor scheme

\section{AIR GAP CORRECTION}

In Fig. 1. the slot rotor pitch (td) and the air-gap $(\delta)$ are constants; in a) the rotor slot width $b$ is constant and in b) the rotor slot width is position dependent $b(x)$. Although the permeability of the gap region is constant, it is bounded on either side by iron surface which far from being smooth, is indented with slots in the circumferential direction, introducing variations in the air gap permeance[6]. It is possible to suppose that the actual slotted surface can be replaced by an equivalent unslotted surface having the same cross-section but with modified "equivalent" air gap $\delta K_{c}$, where $K_{c}$ the so called Carter factor, is the relation between the equivalent air gap permeance and the actual air gap permeance.

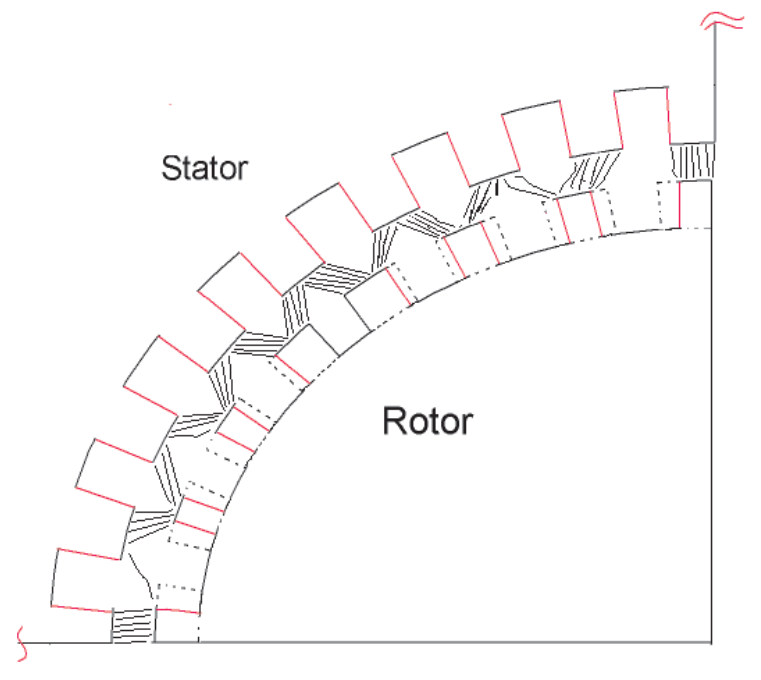

Fig. 2. IMIS stator and rotor scheme.

For the IMIS, the periodical variation of the rotor slot width, superpose a new space variation on the air gap flux density (Fig. 2). It is possible to consider a new correction factor for the sinusoidal modulation of the slot wide rotor, called "Corrected Carter Factor" and it is denoted here as $K_{s}$.

$$
K_{s}=\frac{1}{\alpha_{1}-\alpha_{2} \cos \left(2 \nu \theta_{r}\right)}
$$

where, $\alpha_{1}$ and $\alpha_{2}$ are constants associated with a maximal and minimal air gap equivalent (Fig. 3). The equivalent air gap is:

$$
\begin{aligned}
& \delta_{s}=\delta K_{s}=\delta \frac{1}{\alpha_{1}-\alpha_{2} \cos \left(2 \nu \theta_{r}\right)}=\frac{1}{\delta_{1}-\delta_{2} \cos \left(2 \nu \theta_{r}\right)} \\
& \delta_{1}=\alpha_{1} / \delta \text { and } \delta_{2}=\alpha_{2} / \delta .
\end{aligned}
$$

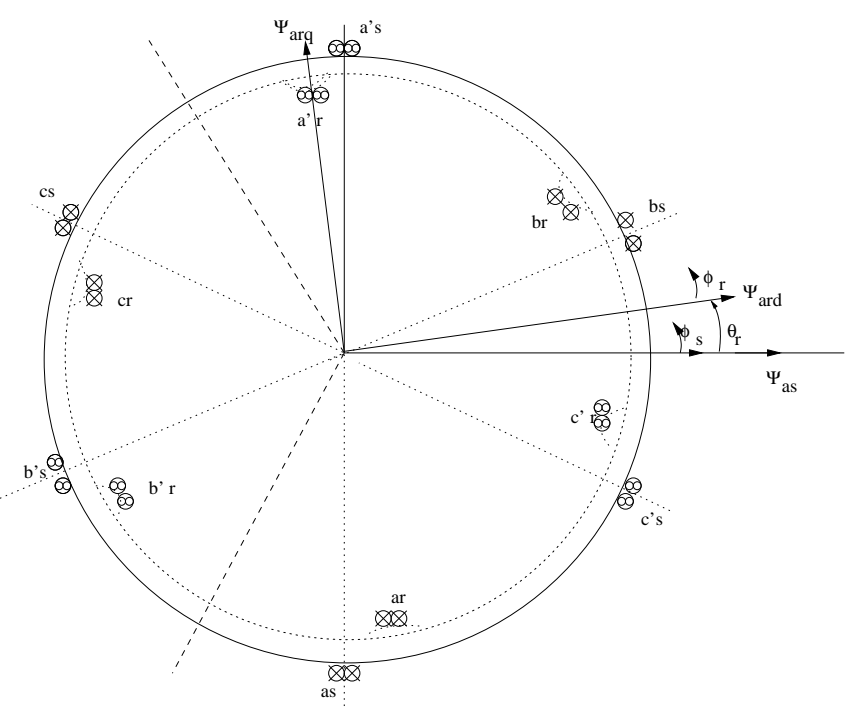

Fig. 3. Scheme of the Induction Motor

Remark: In absence of the rotor slot modulation, $\delta_{2}=$ $\alpha_{2} / \delta=0$, then $\delta_{s}=\delta K_{s}$, so, $K_{s}=K_{c}$ and $\delta_{s}=\delta_{c}$.

\section{WINDING INDUCTANCES}

\section{A. Stator Inductances}

The currents in balanced and steady state conditions are

$$
\begin{aligned}
i_{A} & =\sqrt{2} I_{s} \cos \left(w_{a} t+\phi_{s}(0)\right) \\
i_{B} & =\sqrt{2} I_{s} \cos \left(w_{a} t-2 \pi / 3+\phi_{s}(0)\right) \\
i_{C} & =\sqrt{2} I_{s} \cos \left(w_{a} t+2 \pi / 3+\phi_{s}(0)\right)
\end{aligned}
$$

Then the magnetomotive forces by the stator windings are respectively:

$$
\begin{aligned}
& \mathcal{F}_{A}=i_{A} N_{s} \cos \left(\nu \phi_{s}\right) \\
& \mathcal{F}_{B}=i_{B} N_{s} \cos \left(\nu \phi_{s}-2 \pi / 3\right) \\
& \mathcal{F}_{C}=i_{C} N_{s} \cos \left(\nu \phi_{s}+2 \pi / 3\right)
\end{aligned}
$$

Using the Amper's Law and the new air gap factor, the phase air gap flux densities are:

$$
\begin{aligned}
& B_{A}\left(\nu \phi_{s}, \theta_{r}\right)=\mu_{0} \frac{F_{A}}{\delta_{s}\left(\phi_{s}, \theta_{r}\right)}=\frac{\mu_{0} N_{s}}{\delta_{s}\left(\phi_{s}, \theta_{r}\right)} i_{A} \cos \left(\nu \phi_{s}\right) \\
& B_{B}\left(\nu \phi_{s}, \theta_{r}\right)=\frac{\mu_{0} N_{s}}{\delta_{s}\left(\phi_{s}, \theta_{r}\right)} i_{B} \cos \left(\nu \phi_{s}-2 \pi / 3\right) \\
& B_{C}\left(\nu \phi_{s}, \theta_{r}\right)=\frac{\mu_{0} N_{s}}{\delta_{s}\left(\phi_{s}, \theta_{r}\right)} i_{C} \cos \left(\nu \phi_{s}+2 \pi / 3\right)
\end{aligned}
$$


The phase flux and flux linkages are:

$$
\begin{aligned}
\psi_{s x}\left(\nu \phi_{s}, \theta_{r}\right)= & \int_{\phi_{s}}^{\phi_{s}+2 \pi / 2 \nu} B_{x}\left(\nu \xi, \theta_{r}\right) r l d \xi \\
\Psi_{s x}\left(\nu \phi_{s}, \theta_{r}\right)= & \nu \int N_{x}\left(\phi_{s}\right) \psi_{s x}\left(\nu \phi_{s}, \theta_{r}\right) d \phi_{s} \\
= & \nu \int N_{x}\left(\phi_{s}\right) \int_{\phi_{s}}^{\phi_{s}+2 \pi / 2 \nu} B_{x}\left(\nu \xi, \theta_{r}\right) \\
& \cdot r l d \xi d \phi_{s}
\end{aligned}
$$

where the sub index "x" is for the $A, B$ or $C$ windings; "l" and " $r$ " are respectively, the axial length and the mean radius at the air gap of the machine, " $\xi$ " is the integration variable and $N_{x}\left(\phi_{s}\right)$ represents the sinusoidal distributed winding.

So, to the phase A,

$$
\begin{aligned}
\Psi_{s A}= & \nu \int N_{A}\left(\phi_{s}\right) \int_{\phi_{s}}^{\phi_{s}+\pi / \nu} B_{A}\left(\nu \xi, \theta_{r}\right) r l d \xi d \phi_{s} \\
= & -N_{s}^{2} \mu_{0} \nu i_{A} r l \int_{\pi / \nu}^{2 \pi / \nu} \sin \left(\nu \phi_{s}\right) . \\
& \int_{\phi_{s}}^{\phi_{s}+\pi / \nu} \cos (\nu \xi) \\
& \cdot\left\{\delta_{1}-\delta_{2} \cos 2 \nu\left(\phi_{s}-\theta_{r}\right)\right\} d \xi d \phi_{s}
\end{aligned}
$$

The total phase flux linkages are:

$$
\begin{aligned}
& \Psi_{A}=L_{\sigma s} i_{A}+\Psi_{s A} \\
& \Psi_{B}=L_{\sigma s} i_{B}+\Psi_{s B} \\
& \Psi_{C}=L_{\sigma s} i_{C}+\Psi_{s C}
\end{aligned}
$$

Neglecting the phase leakage inductance $\left(L_{\sigma s}\right)$, the stator inductances are:

$$
\begin{aligned}
& L_{A A}=\frac{N_{s}^{2}}{\nu} \pi \mu_{0} r l\left\{\delta_{1}-\frac{\delta_{2}}{2} \cos \left(2 \nu \theta_{r}\right)\right\} \\
& L_{B B}=\frac{N_{s}^{2}}{\nu} \pi \mu_{0} r l\left\{\delta_{1}-\frac{\delta_{2}}{2} \cos \left(2 \nu \theta_{r}-\frac{2 \pi}{3}\right)\right\} \\
& L_{C C}=\frac{N_{s}^{2}}{\nu} \pi \mu_{0} r l\left\{\delta_{1}-\frac{\delta_{2}}{2} \cos \left(2 \nu \theta_{r}+\frac{2 \pi}{3}\right)\right\}
\end{aligned}
$$

The mutual inductances are calculated following the same procedure; the mutual flux linkages are:

$$
\begin{aligned}
\left.\Psi_{A}\right|_{i_{B}}= & -\nu \int_{\pi / \nu}^{2 \pi / \nu} N_{s} \sin \left(\nu \phi_{s}\right) . \\
& \int_{\phi_{s}}^{\phi_{s}+\pi / \nu} \mu_{0} N_{s} i_{B} \cos \left(\nu \xi-\frac{2 \pi}{3}\right) r l \\
& \cdot\left\{\delta_{1}-\delta_{2} \cos 2 \nu\left(\xi-\theta_{r}\right)\right\} d \xi d \phi_{s}
\end{aligned}
$$

Therefore, the mutual inductances for stator windings are:

$$
\begin{aligned}
& L_{A B}=-\frac{N_{s}^{2}}{2 \nu} \pi \mu_{0} r l \delta_{1}-\frac{N_{s}^{2}}{2 \nu} \pi \mu_{0} r l \delta_{2} \cos \left(2 \nu \theta_{r}-\frac{2 \pi}{3}\right) \\
& L_{A C}=-\frac{N_{s}^{2}}{2 \nu} \pi \mu_{0} r l \delta_{1}-\frac{N_{s}^{2}}{22} \pi \mu_{0} r l \delta_{2} \cos \left(2 \nu \theta_{r}+\frac{2 \pi}{3}\right) \\
& L_{B C}=-\frac{N_{s}^{2}}{2 \nu} \pi \mu_{0} r l \delta_{1}-\frac{N_{s}^{2}}{2 \nu} \pi \mu_{0} r l \delta_{2} \cos \left(2 \nu \theta_{r}\right) \\
& \text { and } L_{B A}=L_{A B}, L_{C A}=L_{A C}, L_{C B}=L_{B C} .
\end{aligned}
$$

If,

$$
L_{s} \quad=\frac{N_{s}^{2}}{\nu} \pi \mu_{0} r l \delta_{1} \quad ; \Delta L_{s} \quad=\frac{N_{s}^{2}}{2 \nu} \pi \mu_{0} r l \delta_{2}
$$

then,

$$
\begin{aligned}
L_{A A} & =\frac{N_{s}^{2}}{\nu} \pi \mu_{0} r l \delta_{1}-\frac{N_{s}^{2}}{2 \nu} \pi \mu_{0} r l \delta_{2} \cos \left(2 \nu \theta_{r}\right) \\
& =L_{s}-\Delta L_{s} \cos \left(2 \nu \theta_{r}\right)
\end{aligned}
$$

So, for the others inductances in the stator

$$
\begin{aligned}
& L_{B B}=L_{s}-\Delta L_{s} \cos \left(2 \nu \theta_{r}-2 \pi / 3\right) \\
& L_{C C}=L_{s}-\Delta L_{s} \cos \left(2 \nu \theta_{r}+2 \pi / 3\right) \\
& L_{A B}=-\frac{L_{s}}{2}-\Delta L_{s} \cos \left(2 \nu \theta_{r}-2 \pi / 3\right) \\
& L_{A C}=-\frac{L_{s}}{2}-\Delta L_{s} \cos \left(2 \nu \theta_{r}+2 \pi / 3\right) \\
& L_{B C}=-\frac{L_{s}}{2}-\Delta L_{s} \cos \left(2 \nu \theta_{r}\right) \\
& L_{C A}=L_{A C} \\
& L_{C B}=L_{B C}
\end{aligned}
$$

finally:

$$
\mathbf{七}_{\mathbf{s s}}=\left[\begin{array}{lll}
L_{A A} & L_{A B} & L_{A C} \\
L_{B A} & L_{B B} & L_{B C} \\
L_{C A} & L_{C B} & L_{C C}
\end{array}\right]
$$

\section{B. Rotor Inductances}

Assuming wound symmetrical currents in the rotor:

$$
\begin{aligned}
i_{a} & =\sqrt{2} I_{r} \cos \left(w_{r} t+\phi_{r}(0)\right) \\
i_{b} & =\sqrt{2} I_{r} \cos \left(w_{r} t-2 \pi / 3+\phi_{r}(0)\right) \\
i_{c} & =\sqrt{2} I_{r} \cos \left(w_{r} t+2 \pi / 3+\phi_{r}(0)\right)
\end{aligned}
$$

Where $w_{r}$ is the angular speed in the rotor.

The direct and quadrature axis components of the "a" phase rotoric current are:

$$
i_{a d}=i_{a} \cos \left(\nu \theta_{r}\right) \quad ; \quad i_{a q}=-i_{a} \sin \left(\nu \theta_{r}\right)
$$

$\theta_{r}$ is the angle between the " $\mathrm{A}$ " stator phase axis and " $\mathrm{a}$ " rotor phase axis.

The flux linkage, created by a direct $\left(i_{a d}\right)$ and quadrature $\left(i_{a q}\right)$ components are respectively:

$$
\psi_{d}^{r}=i_{a d} L_{a d} \quad ; \psi_{q}^{r}=i_{a q} L_{a q}
$$

Assuming non saturation, the total flux linkage for a rotor phase "a" is the addition of the flux linkages $\psi_{d}^{r} \mathrm{y} \psi_{q}^{r}$,

$$
\psi_{r a}=L_{a a} i_{a}=\psi_{d}^{r} \cos \left(\nu \theta_{r}\right)-\psi_{q}^{r} \sin \left(\nu \theta_{r}\right)
$$

and,

$$
\begin{aligned}
L_{a a} & =\frac{\psi_{d}^{r} \cos \left(\nu \theta_{r}\right)-\psi_{q}^{r} \sin \left(\nu \theta_{r}\right)}{i_{a}} \\
& =\frac{i_{a d} L_{a d} \cos \left(\nu \theta_{r}\right)-i_{a q} L_{a q} \sin \left(\nu \theta_{r}\right)}{i_{a}} \\
& =\frac{i_{a} \cos \left(\nu \theta_{r}\right) L_{a d} \cos \left(\nu \theta_{r}\right)+i_{a} \sin \left(\nu \theta_{r}\right) L_{a q} \sin \left(\nu \theta_{r}\right)}{i_{a}} \\
& =L_{a d} \cos ^{2}\left(\nu \theta_{r}\right)+L_{a q} \operatorname{sen}\left(\nu \theta_{r}\right) \\
& =L_{r a}-\triangle L_{r a} \cos \left(2 \nu \theta_{r}\right)
\end{aligned}
$$

with

$$
L_{r a}=\frac{1}{2}\left(L_{a d}+L_{a q}\right) ; \triangle L_{r a}=\frac{1}{2}\left(L_{a q}-L_{a d}\right)
$$

Similarly, to the other phases,

$$
\begin{aligned}
& L_{b b}=L_{r}-\triangle L_{r} \cos \left(2 \nu \theta_{r}+2 \pi / 3\right) \\
& L_{c c}=L_{r}-\triangle L_{r} \cos \left(2 \nu \theta_{r}+4 \pi / 3\right)
\end{aligned}
$$


The mutual inductances $L_{a b}, L_{a c}$, and $L_{b c}$ are calculated in the same way,

$$
\begin{aligned}
L_{b a} & =\frac{\psi_{b a}}{i_{a}}=-\frac{L_{r}}{2}-\triangle L_{r} \cos \left(2 \nu \theta_{r}+4 \pi / 3\right) \\
L_{c a} & =\frac{\psi_{c a}}{i_{a}}=-\frac{L_{r}}{2}-\triangle L_{r} \cos \left(2 \nu \theta_{r}+2 \pi / 3\right) \\
L_{b c} & =\frac{\psi_{b c}}{i_{a}}=-\frac{L_{r}}{2}-\triangle L_{r} \cos \left(2 \nu \theta_{r}\right)
\end{aligned}
$$

and

$$
\mathrm{Ł}_{\mathbf{r r}}=\left[\begin{array}{lll}
L_{a a} & L_{a b} & L_{a c} \\
L_{b a} & L_{b b} & L_{b c} \\
L_{c a} & L_{c b} & L_{c c}
\end{array}\right]
$$

\section{Stator-Rotor Mutual Inductance}

In phase "a" the magnetomotive force in the equivalent winding is:

$$
\mathcal{F}_{a}=N_{r} i_{a} \cos \left(\nu \phi_{r}\right)
$$

Considering the new factor $K_{s}$ and the initial condition $\phi_{s}(0)=0$, the phase "a" flux density distribution is:

$$
B_{a}\left(\nu \phi_{r}, \theta_{r}\right)=\mu_{0} \frac{F_{a}}{\delta_{s}\left(\phi_{s}, \theta_{r}\right)}=\frac{\mu_{0}}{\delta_{s}\left(\phi_{s}, \theta_{r}\right)} N_{r} i_{a} \cos \left(\nu \phi_{r}\right)
$$

The mutual flux between stator phase "A" and rotor phase " $a$ " is then:

$$
\psi_{A a}\left(\nu \phi_{r}, \theta_{r}\right)=\int_{\phi_{s}}^{\phi_{s}+2 \pi / 2 \nu} B_{a}\left(\nu \xi, \theta_{r}\right) r l d \xi
$$

Assuming that the windings have a sinusoidal distribution, the total mutual flux linkage is,

$$
\begin{aligned}
\Psi_{A a}\left(\nu \phi_{r}, \theta_{r}\right)= & -\nu \int_{\pi / \nu}^{2 \pi / \nu} N_{s} \sin \left(\nu \phi_{s}\right) \\
& \cdot \int_{\phi_{s}}^{\phi_{s}+\pi / \nu} \frac{\mu_{0}}{\delta_{s}\left(\phi_{s}, \theta_{r}\right)} N_{r} i_{a} \cos \left(\nu \phi_{r}\right) \\
& \cdot r l d \xi d \phi_{s} \\
= & \frac{N_{s} N_{r}}{\nu} \mu_{0} \pi i_{a} r l\left(\delta_{1}+\frac{\delta_{2}}{2}\right) \cos \left(\nu \theta_{r}\right)
\end{aligned}
$$

and,

$$
L_{A a}=\frac{\Psi_{A a}}{i_{a}}=L_{s r} \cos \left(\nu \theta_{r}\right)
$$

where,

$$
L_{s r}=\frac{N_{s} N_{r}}{\nu} \mu_{0} \pi r l\left(\delta_{1}+\frac{\delta_{2}}{2}\right)
$$

The other mutual inductances are obtained following the same procedure for calculating $L_{A a}$.

$$
\begin{aligned}
& \mathrm{Ł}_{\mathbf{s r}}=L_{s r}\left[\begin{array}{ccc}
\cos (\alpha) & \cos (\alpha+2 \pi / 3) & \cos (\alpha-2 \pi / 3) \\
\cos (\alpha-2 \pi / 3) & \cos (\alpha) & \cos (\alpha+2 \pi / 3) \\
\cos (\alpha+2 \pi / 3) & \cos (\alpha-2 \pi / 3) & \cos (\alpha)
\end{array}\right] \\
& \alpha=\nu \theta_{r}
\end{aligned}
$$

\section{ELECTROMAGNETIC CIRCUITS EQUATIONS}

The three-phase induction motor model with distributed windings, in the estator frame, is

$$
\begin{aligned}
V_{s}^{A B C} & =\mathbf{R}_{\mathbf{s}} i_{s}^{A B C}+\dot{\Psi}_{s}^{A B C} \\
V_{r}^{A B C} & =\mathbf{R}_{\mathbf{r}} i_{r}^{A B C}+\dot{\Psi}_{r}^{A B C}
\end{aligned}
$$

$\mathbf{R}_{\mathbf{s}}$ and $\mathbf{R}_{\mathbf{r}}$ are diagonal matrices in $\mathcal{R}^{3}, V_{s}^{A B C}$ and $V_{r}^{A B C}$ are column vectors describing the stator and rotor voltages respectively.

The flux linkage equations are:

$$
\begin{aligned}
& \Psi_{s}^{A B C}=\mathrm{七}_{\mathbf{s s}} i_{s}^{A B C}+\mathrm{七}_{\mathbf{s r}} i_{r}^{A B C} \\
& \Psi_{r}^{A B C}=\left(\mathrm{七}_{\mathbf{s r}}\right)^{T} i_{s}^{A B C}+\mathrm{七}_{\mathbf{r r}} i_{r}^{A B C}
\end{aligned}
$$

The rotor expressions are referred to the stator frame, using the appropriated transformation.

\section{Electromechanical Equations}

The electromechanical equations are:

$$
\begin{array}{ll}
M \dot{w}_{r} & =-f w+T_{e}-T_{l} \\
\dot{\theta}_{r} & =w_{r}
\end{array}
$$

The electrical torque $T_{e}$ is:

$$
\begin{aligned}
T_{e}= & +\frac{1}{2}\left(i_{s}^{A B C}\right)^{T} \frac{\partial \mathbf{E}_{\mathbf{s s}}}{\partial \theta_{r}} i_{s}^{A B C} \\
+ & \left(i_{s}^{A B C}\right)^{T} \frac{\partial \mathbf{Ł}_{\mathbf{s r}}}{\partial \theta_{r}} i_{r}^{A B C}+\frac{1}{2}\left(i_{r}^{A B C}\right)^{T} \frac{\partial \mathbf{E}_{\mathbf{r r}}}{\partial \theta_{r}} i_{r}^{A B C} \\
& \text { VI. ORTHOGONAL FRAME EQUATIONS }
\end{aligned}
$$

\section{ORTHOGONAL FRAME EQUATIONS}

Due to the polyphasic model complexity, is normal to make simplifications using methods of transformation [8].; the basic Concordia transformation replaces the symmetrical three-phase variables ${ }^{1}$ into symmetrical bi-phase equivalent variables. It is denominated the ortoghonal or "ab" model ${ }^{2}$.

$$
x^{a b h}=\mathbf{T}_{\mathbf{c}} x^{A B C}=\sqrt{2 / 3}\left[\begin{array}{ccc}
1 & -1 / 2 & -1 / 2 \\
0 & \sqrt{3} / 2 & -\sqrt{3} / 2 \\
1 / \sqrt{2} & 1 / \sqrt{2} & 1 / \sqrt{2}
\end{array}\right] x^{A B C}
$$

where:

$x^{A B C} \rightarrow$ three-phase variables; and $x^{a b h} \rightarrow$ orthogonal bi-phase variables.

So, the variables transformations can be expressed according to the relationships in Table I.

Consequently, the mathematical expressions of stator and rotor voltages and flux linkages are:

$$
\begin{aligned}
V_{s}^{a b} & =\mathbf{R}_{\mathbf{s}} i_{s}^{a b}+\dot{\Psi}_{s}^{a b} \\
0 & =\mathbf{R}_{\mathbf{r}} i_{r}^{a b}+\dot{\Psi}_{r}^{a b} \\
\Psi_{s}^{a b} & =\mathbf{L s s}\left(2 \nu \theta_{r}\right) i_{s}^{a b}+\mathbf{L s r}^{T}\left(\nu \theta_{r}\right) i_{r}^{a b} \\
\Psi_{r}^{a b} & =\mathbf{L s r}\left(\nu \theta_{r}\right) i_{s}^{a b}+\mathbf{L r r}\left(2 \nu \theta_{r}\right) i_{r}^{a b}
\end{aligned}
$$

${ }^{1}$ Defined as a set of equal amplitud sisusoidal which are displaced by 120 degress.

${ }^{2}$ Also referenced as "dqo", we use the "abh" to distinguish of others references frames 
TABLE I

TRANSFORMATION RELATIONSHIPS

\begin{tabular}{|l|l|}
\hline Stator & Rotor \\
\hline$i_{s}^{a b h}=\mathbf{T}_{\mathbf{c}} i_{s}^{A B C}$ & $i_{r}^{a b h}=\mathbf{T}_{\mathbf{c}} i_{r}^{A B C}$ \\
$v_{s}^{a b h}=\mathbf{T}_{\mathbf{c}} v_{s}^{A B C}$ & $v_{r}^{a b h}=\mathbf{T}_{\mathbf{c}} v_{r}^{A B C}$ \\
$\Psi_{s}^{a b h}=\mathbf{T}_{\mathbf{c}} \Psi_{s}^{A B C}$ & $\Psi_{r}^{a b h}=\mathbf{T}_{\mathbf{c}} \Psi_{r}^{A B C}$ \\
$i_{s}^{A B C}=\mathbf{T}_{\mathbf{c}}{ }^{-1} i_{s}^{a b h}$ & $i_{r}^{A B C}=\mathbf{T}_{\mathbf{c}}{ }^{-1} i_{r}^{a b h}$ \\
$v_{s}^{A B C}=\mathbf{T}_{\mathbf{c}}{ }^{-1} v_{s}^{a b h}$ & $v_{r}^{A B C}=\mathbf{T}_{\mathbf{c}}{ }^{-1} v_{r}^{a b h}$ \\
$\Psi_{s}^{A B C}=\mathbf{T}_{\mathbf{c}}{ }^{-1} \Psi_{s}^{a b h}$ & $\Psi_{r}^{A B C}=\mathbf{T}_{\mathbf{c}}{ }^{-1} \Psi_{r}^{a b h}$ \\
\hline
\end{tabular}

where

$$
\begin{aligned}
\mathbf{R}_{\mathbf{s}} & =R_{s} \mathbf{I} \in \Re^{2} \\
\mathbf{R}_{\mathbf{r}} & =R_{r} \mathbf{I} \in \Re^{2} \\
\mathbf{L s s}\left(2 \nu \theta_{r}\right) & =\mathbf{T}_{\mathbf{c}} \mathbf{E}_{s s} \mathbf{T}_{\mathbf{c}}{ }^{-1} \\
& =L_{s} \mathbf{I}-\Delta L_{s} \mathbf{U}^{\mathbf{T}}\left(2 \nu \theta_{r}\right) \mathbf{I}^{-} \\
\operatorname{Lrr}\left(2 \nu \theta_{r}\right) & =\mathbf{T}_{\mathbf{c}} \mathbf{E}_{r r} \mathbf{T}_{\mathbf{c}}{ }^{-1} \\
& =L_{r} \mathbf{I}-\Delta L_{r} \mathbf{U}^{\mathbf{T}}\left(2 \nu \theta_{r}\right) \mathbf{I}^{-} \\
\operatorname{Lsr}\left(\nu \theta_{r}\right) & =L_{s r} \mathbf{U}\left(\nu \theta_{r}\right) \\
\operatorname{Lsr}^{T}\left(\nu \theta_{r}\right) & =L_{s r} \mathbf{U}^{\mathbf{T}}\left(\nu \theta_{r}\right)
\end{aligned}
$$

The electromechanical equation is given by (19), and the transformed torque equation is:

$$
\begin{aligned}
T_{e}= & \frac{1}{2}\left(i_{s}^{a b h}\right)^{T} \frac{\partial \mathbf{L}_{\mathbf{s s}}}{\partial \theta_{r}} i_{s}^{a b h} \\
& +\left(i_{s}^{a b h}\right)^{T} \frac{\partial \mathbf{L}_{\mathbf{s r}}}{\partial \theta_{r}} i_{r}^{a b h}+\frac{1}{2}\left(i_{r}^{a b h}\right)^{T} \frac{\partial \mathbf{L}_{\mathbf{r r}}}{\partial \theta_{r}} i_{r}^{a b h}
\end{aligned}
$$

with

$$
\begin{aligned}
& \frac{\partial}{\partial \theta_{r}}\left(\mathbf{T}_{\mathbf{c}} \mathfrak{七}_{s s} \mathbf{T}_{\mathbf{c}}{ }^{-1}\right)=\frac{\partial \mathbf{L}_{\mathbf{s s}}}{\partial \theta_{r}}=2 \nu \Delta L_{s} \mathbf{U}^{\mathbf{T}}\left(2 \nu \theta_{r}\right) \mathbf{I}^{-} \mathbf{J} \\
& \frac{\partial}{\partial \theta_{r}}\left(\mathbf{T}_{\mathbf{c}} \succeq_{r r} \mathbf{T}_{\mathbf{c}}{ }^{-1}\right)=\frac{\partial \mathbf{L}_{r r}^{r}}{\partial \theta_{r}}=2 \nu \Delta L_{r} \mathbf{U}^{\mathbf{T}}\left(2 \nu \theta_{r}\right) \mathbf{I}^{-} \mathbf{J} \\
& \frac{\partial}{\partial \theta_{r}}\left(\mathbf{T}_{\mathbf{c}} \mathbf{七}_{s r} \mathbf{T}_{\mathbf{c}}{ }^{-1}\right)=\frac{\partial \mathbf{L}_{\mathbf{s r}}}{\partial \theta_{r}}=\nu L_{s r} \mathbf{U}^{\mathbf{T}}\left(\nu \theta_{r}\right) \mathbf{J} \\
& T_{e}=\nu c \Delta L_{s}\left(i_{s}^{a b}\right)^{T} \mathbf{U}^{T}\left(2 \nu \theta_{r}\right) \mathbf{I}^{-} \mathbf{J} i_{s}^{a b} \\
& +\nu c L_{s r}\left(i_{s}^{a b}\right)^{T} \mathbf{U}^{\mathbf{T}}\left(\nu \theta_{\mathbf{r}}\right) \mathbf{J} i_{r}^{a b} \\
& +\nu c \Delta L_{r}\left(i_{r}^{a b}\right)^{T} \mathbf{U}^{T}\left(2 \nu \theta_{r}\right) \mathbf{I}^{-} \mathbf{J} i_{r}^{a b}
\end{aligned}
$$

\section{A. State Space Model}

Considering the stator current $i_{s}^{a b}$ and the rotor flux $\Psi_{r}^{a b}$ as state variables, the model in the natural reference frame is calculated. From (24),

$$
i_{r}^{a b}=\mathbf{L r r}^{-1}\left(2 \nu \theta_{r}\right)\left\{\Psi_{r}^{a b}-L_{s r} \mathbf{U}\left(\nu \theta_{r}\right) i_{s}^{a b}\right\}
$$

Replacing (26) in (22)

$$
\dot{\Psi}_{r}^{a b}=\mathbf{R}_{\mathbf{r}} \mathbf{L r r}^{-\mathbf{1}}\left(2 \nu \theta_{r}\right)\left[L_{s r} \mathbf{U}\left(\nu \theta_{r}\right) i_{s}^{a b}-\Psi_{r}^{a b}\right]
$$

To obtain $i_{s}^{a b}$, replacing (26) in (24) and calculating

$$
\dot{i}_{s}^{a b}=-\mathbf{P}_{\sigma}\left(\theta_{r}, w\right) i_{s}^{a b}-\mathbf{Q}_{\sigma}\left(\theta_{r}, w\right) \Psi_{r}^{a b}+U_{s}^{a b}
$$

where:

$$
\begin{aligned}
\mathbf{P}_{\sigma}\left(\theta_{r}, w\right) & =\mathbf{P}\left(\theta_{r}, w\right) / \text { Det }_{\sigma} \\
\mathbf{Q}_{\sigma}\left(\theta_{r}, w\right) & =\mathbf{Q}\left(\theta_{r}, w\right) / \text { Det }_{\sigma} \\
U_{s}^{a b} & =\sigma^{-\mathbf{1}}\left(\nu \theta_{r}\right) V_{s}^{a b}
\end{aligned}
$$

Again, the electromechanical equation is given by (19), and the transformed torque equation is:

$$
\begin{aligned}
T_{e}= & -\nu c_{1} L_{s r}\left(i_{s}^{a b}\right)^{T} \mathbf{J I}^{-} \mathbf{U}\left(2 \nu \theta_{r}\right) . \\
& {\left[2 \Delta L_{r} \mathbf{U}\left(2 \nu \theta_{r}\right)+\Delta L_{s} \mathbf{I}\right] i_{s}^{a b} } \\
& -\nu \Delta L_{r} c_{1} L_{s r}{ }^{-1}\left(\Psi_{r}^{a b}\right)^{T} \mathbf{J I}^{-} \mathbf{U}\left(2 \nu \theta_{r}\right) \Psi_{r}^{a b} \\
& +\nu c_{1}\left(i_{s}^{a b}\right)^{T} . \\
& \left\{L_{r} \mathbf{U}^{\mathbf{T}}\left(\nu \theta_{r}\right) \mathbf{J}+2 \Delta L_{r} \mathbf{J} \mathbf{I}^{-} \mathbf{U}\left(3 \nu \theta_{r}\right)\right\} \Psi_{r}^{a b} \\
& +\nu c_{1} \Delta L_{r}\left(\Psi_{r}^{a b}\right)^{T} \mathbf{J I}^{-} \mathbf{U}\left(3 \nu \theta_{r}\right) i_{s}^{a b}
\end{aligned}
$$

\section{B. Equivalence among models}

If it is considered the case of the "clasical" IM, i.e, without "induced saliencies", the parameters $\Delta L_{s}$ and $\Delta L_{r}$ are adjusted to zero; in this particular situation it is possible to probe that the IMIS converge to the IM model doing the factors $\Delta_{i}$ in the IMIS equal to zero.

$$
\begin{aligned}
\left.\dot{i}_{s}^{a b}\right|_{\Delta L_{s}=\Delta L_{r}=0}= & -\gamma i_{s}^{a b}+\eta\{a \mathbf{I}-\nu w \mathbf{J}\} \mathbf{U}^{\mathbf{T}}\left(\nu \theta_{r}\right) \Psi_{r}^{a b} \\
& +\frac{V_{s}^{a b}}{\sigma_{J} L_{s}} \\
\left.\dot{\Psi}_{r}^{a b}\right|_{\Delta L_{s}=\Delta L_{r}=0}= & -a \Psi_{r}^{a b}+b \mathbf{U}\left(\nu \theta_{r}\right) i_{s}^{a b} \\
\left.T_{e}\right|_{\Delta L_{s}=\Delta L_{r}=0}= & \nu \frac{L_{s r}}{L_{r}}\left(i_{s}^{a b}\right)^{T} \mathbf{U}^{\mathbf{T}}\left(\nu \theta_{r}\right) \mathbf{J} \Psi_{r}^{a b} \\
\dot{\theta_{r}}= & w
\end{aligned}
$$

Which is the classical $a b$ model found in the induction motors literature[8].

\section{CONCLUSIONS}

A new general model to the IMIS was developed which is computed in natural orthogonal components. The model is suitable for control design and compatible with the classical $a b$ model for induction motors without saliencies. A similar model for control by signals injection at high frequencies was reported by Jansen et al.[4], but the model for the IMIS presented here is more general.

It is observed a complexity in the components of the currents by the presence of the $\Delta_{i}$ variations and their implicit dependence with the rotor position $\theta_{r}$. Note that the rotor position dependence in the model can not be avoided by rotor variables transformations ( $\alpha-\beta$ model).

Current work is to research the stability and observability properties of this model, analyze the induced harmonics in the torque and currents, among others.

\section{REFERENCES}

[1] J. Holtz, "Sensorless control of induction motors-performance and limitations", ISIE 2000, Proceedings of the 2000 IEEE International Symposium on Industrial Electronics, vol. 1, pp PL12 - PL20, 4-8, Dec. 2000. 
[2] J. Holtz, "Sensorless control of induction motors drives", Proceedings of the IEEE, volume = 90, Issue 8, pp. 1359-1394, August 2002.

[3] Michael W. Degner and Robert D. Lorentz, "Using Multiple Saliencies for the estinmations of Flux, Position, and Velocity in AC Machines", IEEE Transaction on Industry Applications, Vol 34, No. 5, September/October 1998.

[4] Patrick Jansen and Robert Lorenz, "Transducerless Position and Velocity Estimation in Induction and Salient AC Machines", IEEE Transaction on Industry Applications, Vol 31, No. 2, March/April 1995.

[5] J. Holtz and Quan,IEEE Industry Applications Soc. Annu. Meeting, Chicago, IL, Sept.30-oct.4, 2001.

[6] T. A. Lipo, "Introduction to AC MAchine Design", Wisconsin Power Electronics Research Center of the University of Wisconsin, Copyright 2000.

[7] F. Briz, M.W. Degner, A. Diez and R. Lorentz, "Measuring, Modeling, and Decoupling of Saturation-Induced Saliencies in Carrier-Signal Injection-Based Sensorless AC Drives", IEEE Transactions on Industry Applications, vol 37, number 5, pp. "1356-1364", September 2001.

[8] Paul C. Krause,Mc. Graw Hill Book Company, Analysis of Electrical MAchinery, Copyrigh 1986, ISBN 0-07-035436-7

\section{APPENDIX}

Definitions and properties

$$
\mathbf{U}(\cdot)=\left[\begin{array}{cc}
\cos (\cdot) & \sin (\cdot) \\
-\sin (\cdot) & \cos (\cdot)
\end{array}\right]
$$

$$
\begin{gathered}
\mathbf{I}=\left[\begin{array}{ll}
1 & 0 \\
0 & 1
\end{array}\right] \quad ; \quad \mathbf{I}^{-}=\left[\begin{array}{cc}
1 & 0 \\
0 & -1
\end{array}\right] \quad ; \quad \mathbf{J}=\left[\begin{array}{cc}
0 & -1 \\
1 & 0
\end{array}\right] \\
\mathbf{U}^{\mathbf{T}}(\cdot)=\mathbf{U}(-(\cdot))
\end{gathered}
$$

$$
\mathbf{I}^{-} \mathbf{I}^{-}=\mathbf{I} \quad ; \quad \mathbf{J J}=\mathbf{I}^{-} \quad ; \quad \mathbf{I}^{-}=\mathbf{I}^{--1}
$$

$$
\mathbf{J I}^{-}=-\mathbf{I}^{-} \mathbf{J}=\mathbf{I}^{-} \mathbf{J}^{\mathbf{T}}=\left[\begin{array}{ll}
0 & 1 \\
1 & 0
\end{array}\right]
$$

To the IM,

$$
\begin{aligned}
\sigma_{J} & \doteq 1-\frac{L_{s r}^{2}}{L_{s} L_{r}} \\
a & \doteq R_{r} / L_{r} \\
\gamma & \doteq \frac{R_{s}}{L_{s} \sigma_{J}}+\frac{R_{r}}{L_{r}^{2}} \frac{L_{s r}^{2}}{L_{s} \sigma_{J}} \\
\eta & \doteq \frac{L_{s r}}{L_{s} L_{r} \sigma_{J}} \\
b & =a L_{s r}
\end{aligned}
$$

To the IMIS,

$$
\begin{array}{ll}
L_{r} & \equiv \frac{L_{r d}+L_{r q}}{L_{r q}-L_{r d}} \\
\Delta L_{r} & \equiv L_{r}-\Delta L_{r} \\
L_{r d} & \equiv L_{r}+\Delta L_{r} \\
L_{r q} & \equiv \frac{L_{s d}+L_{s q}}{L_{s q}-L_{s d}} \\
L_{s} & \equiv L_{s}-\Delta L_{s} \\
\Delta L_{s} & \equiv L_{s}+\Delta L_{s} \\
L_{s d} & \equiv L_{r}^{2}-L_{r q}^{2} \\
L_{s q} & \equiv L_{s}^{2}-L_{s q}^{2} \\
L_{r d} L_{r q} & =L_{s r} /\left(L_{r d} L_{r q}\right) \\
L_{s d} L_{s q} & =L_{s}-c_{1} L_{s r} L_{r} \\
c_{1} & =
\end{array}
$$

$$
\begin{aligned}
& L_{L}=L_{s}-c_{1} L_{s r} L_{r}=L_{s}-\frac{L_{s r}}{L_{r d} L_{r q}} L_{s r} L_{r} \\
& P\left(\theta_{r}, w\right) \quad=\left\{R_{s}+c_{1}^{2}\left(L_{r}^{2}+\Delta L_{r}^{2}\right) R_{r}\right\} \mathbf{I} \\
& +2 L_{r} \Delta L_{r} \mathbf{U}^{T}\left(4 \nu \theta_{r}\right) \mathbf{I}^{-} \\
& -2 \nu w\left(\Delta L_{s} \mathbf{I}+2 c_{1} L_{s r} \Delta L_{r} \mathbf{U}^{T}\left(2 \nu \theta_{r}\right)\right) \\
& \text {. } \mathbf{U}^{T}\left(2 \nu \theta_{r}\right) \mathbf{J I}^{-} \\
& Q\left(\theta_{r}, w\right) \quad=-R_{r} c_{1}^{2}\left(L_{s r}^{-1}\right)\left(L_{r}^{2}+\Delta L_{r}^{2}\right) \mathbf{U}^{\mathbf{T}}\left(\nu \theta_{r}\right) \\
& -2 R_{r} c_{1}^{2}\left(L_{s r}\right)^{-1} L_{r} \Delta L_{r} \mathbf{U}^{\mathbf{T}}\left(3 \nu \theta_{r}\right) \mathbf{I}^{-} \\
& +\nu w\left[c_{1} L_{r} \mathbf{U}^{\mathbf{T}}\left(\nu \theta_{r}\right) \mathbf{J}\right. \\
& \left.+3 c_{1} \Delta L_{r} \mathbf{U}^{\mathbf{T}}\left(3 \nu \theta_{r}\right) \mathbf{J I}^{-}\right] \\
& \boldsymbol{\sigma}^{-\mathbf{1}}\left(\nu \theta_{r}\right) \quad=\frac{1}{\operatorname{Det}_{\sigma}}\left\{L_{L} \mathbf{I}+\Delta L_{s} \mathbf{U}^{\mathbf{T}}\left(2 \nu \theta_{r}\right) \mathbf{I}^{-}\right. \\
& \left.+c_{1} L_{s r} \Delta L_{r} \mathbf{U}^{\mathbf{T}}\left(4 \nu \theta_{r}\right) \mathbf{I}^{-}\right\} \\
& \operatorname{Det}_{\sigma}=\left(L_{L}\right)^{2}-\Delta L_{s}^{2}-c_{1}^{2} L_{s r}^{2} \Delta L_{r}^{2} \\
& -2 c_{1} L_{s r} \Delta L_{s} \Delta L_{r} \cos \left(2 \nu \theta_{r}\right) \\
& \mathbf{L r r}^{-\mathbf{1}}\left(2 \nu \theta_{r}\right)=\frac{1}{L_{r d} L_{r q}}\left(L_{r} \mathbf{I}+\Delta L_{r} \mathbf{U}^{\mathbf{T}}\left(2 \nu \theta_{r}\right) \mathbf{I}^{-}\right)
\end{aligned}
$$

